\title{
London Deauville Criteria Point Scale 5
}

National Cancer Institute

\section{Source}

National Cancer Institute. London Deauville Criteria Point Scale 5. NCI Thesaurus. Code C99750.

The results of the positron emission tomography are positive; the tracer uptake is markedly increased at any disease site. 